\title{
Evolving Dynamics of Trading Behavior based on Coordination Game in Complex Networks
}

\author{
Yue-tang Bian ${ }^{*}, \mathrm{a}, \mathrm{Lu} \mathrm{Xu}^{\mathrm{b}}$, Jin-sheng $\mathrm{Li}^{\mathrm{a}}$ \\ ${ }^{a}$ School of Business, Nanjing Normal University, Nanjing, Jiangsu 210023, China \\ ${ }^{b}$ Nanjing Institute of Railway Technology, Nanjing, Jiangsu 210031, China
}

\begin{abstract}
A B S T R A C T
This work concerns the modeling of evolvement of trading behavior in stock markets. Based on the assumption of the investors' limited rationality, the evolution mechanism of trading behavior is modeled according to the investment strategy of coordination game in network, that investors are prone to imitate their neighbors' activity through comprehensive analysis on the risk dominance degree of certain investment behavior, the network topology of their relationship and its heterogeneity. We investigate by mean-field analysis and extensive simulations the evolution of investors' trading behavior in various typical networks under different risk dominance degree of investment behavior. Our results indicate that the evolution of investors' behavior is affected by the network structure of stock market and the effect of risk dominance degree of investment behavior; the stability of equilibrium states of investors' behavior dynamics is directly related with the risk dominance degree of some behavior; connectivity and heterogeneity of the network plays an important role in the evolution of the investment behavior in stock market.
\end{abstract}

Keywords:

Trading behavior

Evolution model

Coordination game

Complex networks

\section{Introduction}

In behavioral finance literature, investors are considered to be limited rational, especially for the less sophisticated ones, who always attempt to mimic financial gurus or follow the activities of successful investors, since using their own information/knowledge might incur a higher cost [1]. The most typical example is that during the financial crisis, agents were rushed to sell shares in the same direction, leading the market behavior to herding critically. More imitation behavior among the investors is probe to result in herding behavior in financial market, as Nofsinger and Sias [2] note, "a group of investors trading in the same direction over a period of time", and introducing big fluctuations easily, particularly in bull or bear states. Therefore, Mechanism of dynamic and herding behavior of stock markets has attracted much academic and industrial attention.

In recent years, there have been numerous empirical and theoretical studies on the herding behavior phenomenon. The literature about herding behavior phenomenon of stock markets can be classified into two categories. One category focuses on the empirical investigations of herding behavior in financial markets.

In [3], the authors conclude that the nature of herding differs across exchanges and is not universal. Specifically, in emerging markets investors exert herding patterns different from those in developed countries. Zhou and Lai [4] discover that herding activity in Hong Kong's market tends to be more prevalent with small stocks and that investors are more likely to herd when selling rather than buying stocks. In [5], Chiang and Zheng extend the investigation of herding behavior from domestic markets to global markets and find evidence of herding in advanced stock markets (except the USA) and in Asian markets. Turing to the Chinese markets, Demirer and Kutan[6] investigate whether investors in Chinese markets, in making their investment decisions, are following market consensus rather than private information during periods of market stress. Tan et al. [7] report that herding occurs under both rising and falling market conditions and is especially present in A-share investors. In all cases, herding behavior is proved to be a common state of stock behavior's evolving into.

The other one focuses on the evolution dynamics of trading behavior in financial market. By developing a Cellular Automata model of investment behavior in the stock market, Wei et al. find that

\footnotetext{
* Corresponding author.

E-mail address: nnubianyt@163.com (Y. Bian)
} 
increased imitation among investors leads to a less stable market [8]. In [9], Liang and Han find that evolvement of investors' trading behavior in stock market emerges most of stylized facts, such as clustered volatility, bubbles and crashes, in artificial stock market. By incorporating stock price into investor decisions, L. Bakker et al. construct a social network model of investment behavior in stock market and find that real life trust networks can significantly delay the stabilization of a market [10]. Chen et al. show that stock price bubbles or crashes are caused by synergy herding behavior though imitation agent and market sentiment signals [11]. Falbo and Grassi propose a market with two kinds of agents: speculators and rational investors to analyze the dynamics of a financial market when agents anticipate the occurrence of a correlation breakdown, and finds that the market equilibrium results depend on the prevalence of one of the two types of agents [12]. Consequently, it has a great important chance to study the evolvement of the trading behavior, for it affects the market critically and vice versa.

All the above-mentioned models and methods have captured some feature of investor trading behavior and its impact on the market, but most of the studies mainly focus on the macro level of trading behavior through quantitative analysis. This paper differs from previous research in the following respects. In this work, the evolution mechanism of individual investors' trading behavior and its collective dynamics is investigated from microscopic perspective by the theory and methods in the field of complexity science. Meanwhile, all traders around the world can be seen as a network organized from family, friends, colleagues, incorporated not only by the source of opinion but also the local influence among them [15]. We consider a network of interacting agents whose trading behavior is determined by the action of their peer neighbors, according to the payoff of using coordination game strategy. By means of mean-field approach and simulation analysis, the evolvement equilibrium of investors' trading behavior is analyzed and it crucially depends on two components: the connectivity distribution of the network and the risk dominance degree of a certain investment behavior, which play an important role in the stability of equilibrium states of investors' behavior dynamics.

The rest of the paper is organized as follows. In Section 2, we introduce the model and define the evolvement rules of investor's trading behavior. In Section 3, the evolvement characteristics of the trading behavior are presented by the method of mean-field equation. In Section 4, the comparison between analytic and simulation results are conducted. And the conclusion is drawn in Section 5.

\section{The Model}

\subsection{The network}

Due to the difficulties of finding the relevant data to determine the network of exposures, financial network theory has been forced to either use important assumptions (like maximum entropy) or to resort to different theories and tools, like random graphs (see Erdös and Rényi, 1959) or scale-free networks (see Barabási and Albert, 1999). Random graphs are important because most network formation models are based on variations of random graphs. On the other hand, scale-free networks are described in a simplistic way as networks in which the distribution of the nodes' degree follows a power law distribution. Scale-free networks are a popular subject of study as many of the well-known networks possess this property (for example, the WWW, Wikipidia and the citation networks).

Nature, society, and many technologies are sustained by numerous networks that are not only too important to fail but paradoxically for decades have also proved too complicated to understand [14]. Based on the viewpoint posted by Johansen and Sornette in [13], the evolution system of investors' behavior in stock markets can be described as a network, while nodes represent investors, the edges between every two nodes represent their relationship, such as, social relations, trade association, or something.

Consider a finite but large population of individuals $N=\{1,2, \ldots, i, n\}$. Each investor $i \in N$ interacts with a subset of the population which form a complex network $G=(N, V)$, where $(i, j) \in V$ means that $i$ and $j$ are linked in network. We consider undirected networks, i.e., if $(i, j) \in V$ then $(j, i) \in V$. Let $N_{i}$ be the set of individuals with whom $i$ is linked. Formally, $N_{i}=\{j \in N$, s.t. $(i, j) \in L\}$, where $k_{i}=\left|N_{i}\right|$ is the number of neighbors of $i$, often referred as his connectivity. The connectivity can differ across individuals in the population and its distribution $P(k)$ displays for each $k \geq 1$ the fraction of nodes with connectivity $k$. More precisely, $P(k)=1 / n \mid\left\{i \in N\right.$, s.t. $\left.k_{i}=k\right\} \mid$.

\subsection{The evolvement rule}

Our model focuses evolvement of trading behavior in a population of stock markets. Normally, a trader $i \in N$ can only has three discreet states: $s_{i} \in\{-1,0,1\}$, where $s_{i}=-1$ if $i$ select behavior "sell", $s_{i}=1$ 
if $i$ select behavior "buy", and $s_{i}=0$ if $i$ select behavior "hold". At time $t$, considering the following continuous dynamic process, the state of the stock market is a vector $s_{t}=\left(s_{1 t}, s_{2 t}, \ldots, s_{i t}, \ldots, s_{N t}\right) \in S^{N} \equiv\{-1,0,1\}^{N}$. So, each investor can switch their trading behavior among these three states.

Supposing that all investors' initial investment behavior is "hold", there will be three options for an investor to make a choice in the next time step whose behavior is holding the line, while there will be alternative of behavior "buy" and "sell" in the next time step for investor whose behavior has been changed.

For investors being limited rational and their incompletely know about the market information, the only information, except for the public ones, an investor can get is from his neighbors' return status, so the limited rational investors are about to have "strategic imitation" behavior with cooperative feature. It is said that the investors make their own investment decisions using the cooperative game strategy. Based on the investment behavior's state space $S=\{1,0,-1\}^{N}$, every investor plays $3 \times 3$ game with his neighbors at each time $t$, and obtain his benefits according to his payoff function $\pi\left(s, s^{\prime}\right)$, where $s, s^{\prime} \in S$. Table 1 is the payoff matrix of coordination game.

Table 1. Payoff Matrix of $3 \times 3$ Coordination Game.

\begin{tabular}{|c|c|c|c|c|}
\hline & \multicolumn{4}{|c|}{ Investor $j$} \\
\hline & & Strategy $s_{j}=1$ & Strategy $s_{j}=0$ & Strategy $s_{j}=-1$ \\
\hline \multirow[t]{3}{*}{ Investor $i$} & Strategy $s_{i}=1$ & $\pi(1,1) \quad \pi(1,1)$ & $\pi(1,0) \quad \pi(0,1)$ & $\pi(1,-1) \quad \pi(-1,1)$ \\
\hline & Strategy $s_{i}=0$ & $\pi(0,1) \quad \pi(1,0)$ & $\pi(0,0) \quad \pi(0,0)$ & $\pi(0,-1) \quad \pi(-1,0)$ \\
\hline & Strategy $s_{i}=-1$ & $\pi(-1,1) \quad \pi(1,-1)$ & $\pi(-1,0) \quad \pi(0,-1)$ & $\pi(-1,-1) \quad \pi(-1,-1)$ \\
\hline
\end{tabular}

Let $\pi(1,1)>\pi(0,1), \pi(1,1)>\pi(-1,1) \quad, \pi(0,0)>\pi(1,0) \quad, \pi(0,0)>\pi(-1,0) \quad, \pi(-1,-1)>\pi(1,-1)$ and $\pi(-1,-1)>\pi(0,-1)$, Table 1 shows a coordination game, and has the Nash Equilibrium of $[\pi(1,1), \pi(1,1)] 、[\pi(0,0), \pi(0,0)]$ and $[\pi(-1,-1), \pi(-1,-1)]$. Thus, at any time, investor $i$ 's total return is the sum of game payoff with its neighbors.

Based on the above analysis, investor $i$ can only make his decision according to his neighbors' behavior at last moment, so as to make his own benefit expectation maximum at this moment, that is to say, investors are about to make short-term optimal reaction to the last payoff matrix. Thus, at any time, if the proportion of investors who select behavior "buy" exceed $q_{1}$, behavior "buy" is the optimal reaction strategy; if the proportion of investors who select behavior "sell" exceed $q_{2}$, behavior "sell" is the optimal reaction strategy; otherwise, behavior "hold" is the optimal reaction strategy, and then the proportion of investors who select behavior "hold" exceed $q_{3}$, where $q_{3}=1-q_{1}-q_{2}$. Table 2 shows the modified payoff matrix of coordination game.

Table 2. Modified Payoff Matrix of $3 \times 3$ Coordination Game.

\begin{tabular}{|c|c|c|c|c|}
\hline & \multicolumn{4}{|c|}{ Investor $j$} \\
\hline \multirow{4}{*}{ Investor $i$} & & Strategy $s_{j}=1$ & Strategy $s_{j}=0$ & Strategy $s_{j}=-1$ \\
\hline & Strategy $s_{i}=1$ & $q_{1} \quad q_{1}$ & 00 & 00 \\
\hline & Strategy $s_{i}=0$ & $0 \quad 0$ & $1-q_{1}-q_{2} \quad 1-q_{1}-q_{2}$ & 00 \\
\hline & Strategy $s_{i}=-1$ & 00 & 00 & $q_{2} \quad q_{2}$ \\
\hline
\end{tabular}

Based on the evolution rules of investors' behavior under the coordination game strategy and the principle of risk dominance, let $q_{1}$ be the risk dominance degree of behavior "buy", the less $q_{1}$, the higher risk dominance degree of behavior "buy" and the investors prefer this behavior. Obviously, $q_{1}$ depends on the income of investment. Considering that investors are about to modify their 
investment decision frequently as the stock market volatility and supposing that risk dominance degree of behavior "sell" is always less than behavior "hold" in the mixed Nash Equilibrium of Coordination game, the mean field equation can be used to analyze the dynamic evolution characteristics of herding behavior under the coordination game strategy in network.

\section{Mean-field analysis}

Supposing the network size is large enough and all the nodes being homogeneous in mixture distribution, dynamic evolution characteristics can be analyzed by mean-field equation on the condition that each node's property in investment network is not affected by network topology. Let $\rho_{1, k}(t)$ be the relative density of trader nodes in network who show trading behavior "buy" at time $t$ with connectivity $k$ and $\frac{k P(k)}{\langle k\rangle}$ be the possibility that any given link points to a trader node with connectivity $k$ where $<k>=\sum_{k \geq 1}^{\prime} k P(k)$, so at any time $t$, the possibility that any given link points to a trader point with behavior "buy" can be defined as $\theta_{1}(t)=\frac{\sum_{k \geq 1} k P(k) \rho_{1, k}(t)}{<k>}$. Thus, the probability that a "none-buy" behavior trader with $k$ neighbors has exactly $k_{1}$ neighbors with "buy" behavior at time $t$ is $\left(\begin{array}{l}k \\ k_{1}\end{array}\right) \theta_{1}^{k_{1}}\left(1-\theta_{1}\right)^{k-k_{1}}$. Denote by $\rho_{-1, k}(t)$ and $\rho_{0, k}(t)$ the proportion of the nodes with connectivity $k$ who select behavior "sell" and "hold" at time $t$, the possibility that any given link points to a trader point with behavior "sell" and "hold" is $\theta_{-1}(t)=\frac{\sum_{k \geq 1} k P(k) \rho_{-1, k}(t)}{<k>}$ and $\theta_{0}(t)=\frac{\sum_{k \geq 1} k P(k) \rho_{0, k}(t)}{<k>}$ respectively at time $t$. In the same, the probability that a trader with $k$ neighbors has exactly $k_{2}$ neighbors with "sell" behavior and $k_{3}$ neighbors with "hold" behavior at time $t$ is $\left(\begin{array}{c}k \\ k_{2}\end{array}\right) \theta_{-1}{ }^{k_{2}}\left(1-\theta_{-1}\right)^{k-k_{2}}$ and $\left(\begin{array}{c}k \\ k_{3}\end{array}\right) \theta_{0}^{k_{3}}\left(1-\theta_{0}\right)^{k-k_{3}}$, where $k_{1}+k_{2}+k_{3}=k$.

On this basis, the possibility that one trader with $k$ neighbors, who selecting behavior "buy", of whom $k_{1}$ are with behavior "buy", can be defined as $P_{q_{1}}\left(1 \mid k_{1}, k\right)$; while, the possibility that one trader with $k$ neighbors, who selecting behavior "sell", of whom $k_{2}$ are with behavior "sell" , can be defined as $P_{q_{2}}\left(-1 \mid k_{2}, k\right)$; and the possibility that one trader with $k$ neighbors, who selecting behavior "hold", of whom $k_{3}$ are with behavior "hold", is $1-P_{q_{1}}\left(1 \mid k_{1}, k\right)-P_{q_{2}}\left(-1 \mid k_{2}, k\right)$. Supposing that investors select the short-term determining optimal response strategy, the possibility that investors selecting some behavior depends on the relationship between the risk dominance degree of mixed strategy Nash Equilibrium and the proportion of some behavior which the neighbor nodes select in coordination game. If and only if $k_{1} / k>q_{1}, P_{q_{1}}\left(1 \mid k_{1}, k\right)=1$ and when $k_{2} / k>q_{2}, P_{q_{2}}\left(-1 \mid k_{1}, k\right)=1$, otherwise, $\quad P_{q_{1}}\left(1 \mid k_{1}, k\right)=P_{q_{2}}\left(-1 \mid k_{1}, k\right)=0$. If two of the following expression, such as $k_{3} / k>1-P_{q_{1}}\left(1 \mid k_{1}, k\right)-P_{q_{2}}\left(-1 \mid k_{1}, k\right) 、 k_{1} / k>q_{1}$ 和 $k_{2} / k>q_{2}$, or all the three are tenable, the behavior who has the lowest risk dominance degree will be selected.

Let $\lambda>0$ be the possibility that an investor adjust his behavior, so the possibility that an investor select behavior "buy" can be denoted by rate $\left(1 \mid k, \theta_{1}(t)\right)=\sum_{k_{1}=0}^{k} \lambda P_{q_{1}}\left(1 \mid k_{1}, k\right)\left(\begin{array}{c}k \\ k_{1}\end{array}\right) \theta_{1}^{k_{1}}\left(1-\theta_{1}\right)^{k-k_{1}}$. In the same, the possibility that an investor select behavior "sell" can be denoted 
by rate $\left(-1 \mid k, \theta_{-1}(t)\right)=\sum_{k_{2}=0}^{k} \lambda P_{q_{2}}\left(-1 \mid k_{2}, k\right)\left(\begin{array}{c}k \\ k_{2}\end{array}\right) \theta_{-1}{ }^{k_{2}}\left(1-\theta_{-1}\right)^{k-k_{2}}$. Thus, the possibility of selecting behavior "hold" is $\lambda$-rate $\left(1 \mid k, \theta_{1}(t)\right)-$ rate $\left(-1 \mid k, \theta_{-1}(t)\right)$. By mean of Mean-field equation, the change rate of an investor selecting behavior "buy" in network can be explained as:

$$
\frac{d \rho_{1, k}(t)}{d t}=\left(\rho_{-1, k}(t)+\rho_{0, k}(t)\right) \operatorname{rate}\left(1 \mid k, \theta_{1}(t)\right)-\rho_{1, k}(t) \operatorname{rate}\left(-1 \mid k, \theta_{-1}(t)\right),
$$

where $\operatorname{rate}\left(0 \mid k, \theta_{0}(t)\right)=1-\operatorname{rate}\left(1 \mid k, \theta_{1}(t)\right)-\operatorname{rate}\left(-1 \mid k, \theta_{-1}(t)\right)$.

Expression (2) shows the flowing, the variation of the relative density of "buy" behavior traders with $k$ links at time $t$ equals the proportion of "non-buy" behavior traders with $k$ neighbors at time $t$ who change their behavior minus the proportion of "buy" behavior traders with $k$ neighbors at time $t$ who become "non-buy" behavior ones. Thus, the variation of the relative density of "buy" behavior traders depends on the variation of the investor's behavior and the behavior status at the right time step, being irrelevant with the time, the variation of the investor's behavior can be seen as a markov progress of continuous time system. Let $\frac{d \rho_{1, k}(t)}{d t}=0$, the proportion of investors who select behavior "buy" at the equilibrium state can be obtained as:

$$
\rho_{1, k}(t)=\frac{1}{\lambda\left(1-\operatorname{rate}\left(0 \mid k, \theta_{0}(t)\right)\right.} \operatorname{rate}\left(1 \mid k, \theta_{1}(t)\right) .
$$

Considering the stock market's volatility, the amount of investors who selecting behavior "hold" is going to be in a small scale which becomes relatively stable within expected time and gradually tends to zero in the evolution process. Based on this, assuming that the change rate of the investor's modifying the behavior "hold" goes to $\operatorname{rate}\left(0 \mid k, \theta_{0}(t)\right) \rightarrow 0$, the proportion of investors' selecting behavior "buy" can be written as:

$$
\rho_{1, k}(t) \simeq \frac{1}{\lambda} \operatorname{rate}\left(1 \mid k, \theta_{1}(t)\right)
$$

Let equation (4) substituted into $\theta_{1}(t)=\frac{\sum_{k \geq 1} k P(k) \rho_{1, k}(t)}{<k>}$, we can obtain that $\theta_{1}(t)=H_{q_{1}}\left(\theta_{1}\right)$, that is the un-trivial solution of $\theta_{1}$ in equilibrium, where $H_{q_{1}}\left(\theta_{1}\right)=\frac{\sum_{k \geq 1} k P(k)}{<k>} \sum_{k_{1}=0}^{k} P_{q_{1}}\left(1 \mid k_{1}, k\right)\left(\begin{array}{c}k \\ k_{1}\end{array}\right) \theta_{1}^{k_{1}}\left(1-\theta_{1}\right)^{k-k_{1}}$. Thus, the proportion of investors' selecting behavior "buy" in equilibrium with the time evolution can be written as $\rho=\sum_{k \geq 1} P(k) \rho_{1, k}$.

Based on the above analysis, according to the mechanism of coordination game and the degree distribution of stock network and if there is only a few investors whose initial behavior is "buy", the equilibrium state of investors' selecting behavior "buy" must be achieved. The proof as follows:

Proof: the equilibrium proportion of the investors' selecting behavior "buy" under the coordination game investment strategy can be shown as:

$$
\theta_{1}=H_{q_{1}}\left(\theta_{1}\right)=\frac{\sum_{k \geq 1} k P(k)}{<k>} \sum_{k_{1}=0}^{k} P_{q_{1}}\left(1 \mid k_{1}, k\right)\left(\begin{array}{c}
k \\
k_{1}
\end{array}\right) \theta_{1}^{k_{1}}\left(1-\theta_{1}\right)^{k-k_{1}} .
$$

Assuming that behavior "buy" is risk dominant, for $k_{1} / k>q_{1}, P_{q_{1}}\left(1 \mid k_{1}, k\right)=1$; otherwise, $P_{q_{1}}\left(1 \mid k_{1}, k\right)=0$. Thus, equation (5) can be shown as: 


$$
\theta_{1}=H_{q_{1}}\left(\theta_{1}\right)=\frac{\sum_{k \geq 1} k P(k)}{<k>} \sum_{k_{1}=\left[k k_{1}\right]+1}^{k}\left(\begin{array}{c}
k \\
k_{1}
\end{array}\right) \theta_{1}^{k_{1}}\left(1-\theta_{1}\right)^{k-k_{1}}
$$

Where $\left[k q_{1}\right]$ represents the integer part of $k q_{1}$. From equation (6), when $q_{1} \in[0,1]$, there exists $H_{q_{1}}(0)=0$ and $H_{q_{1}}(1)=1$, therefore, there's certainly the possibility that $\theta^{*} \in[0,1]$, that is the possibility of the stable equilibrium state of investors' behavior "buy" in network.

\section{Comparison between analytic and simulation results}

Under the strategy of coordination game in network, investment behavior risk dominance degree $q$ and network topology structure $P(k)$ directly affect the evolution of herding behavior in stock market. In this section, we will present the analytic and simulation results so as to study how the connectivity distribution and the evolvement mechanism affect the mean-field equilibrium outcomes of the trading behavior in stock markets.

\subsection{Influence of investment strategy risk dominance on the evolution of herding behavior}

On the basis of the above analysis, assuming that $H_{q_{1}}(\theta)$ is monotonic in the domain of definition, that is to say, we can establish an environment of $H_{q_{1}}^{\prime}(\theta)>=0$. Considering that the values of $\left[k q_{1}\right]$ in the same interval are equal, for example: when $q_{1} \in(0,1 / k),\left[k q_{1}\right]=0$; when $q_{1} \in[1 / k, 2 / k)$, $\left[k q_{1}\right]=1 ; \ldots \ldots$; when $q_{1} \in[(k-1) / k, 1),\left[k q_{1}\right]=k-1$. So, $\left[k q_{1}\right]$ increases in line with $q_{1}$. Based on this, the analysis on the analytic and simulation of behavior "buy" risk dominance degree's impact on the evolution of investment behavior in the same interval and different interval respectively are as follows.

\subsubsection{Theoretical analysis}

( i ) Effect of risk dominant degree in the same interval

As for the risk dominance degree $q_{1}^{\prime}$ and $q_{1}^{\prime \prime}$ of the investment behavior "buy" in the same interval, supposing that the two degrees has the following relationship:

$$
\begin{gathered}
\left\{\begin{array}{l}
\left(k^{\prime}+1\right) / k>q_{1}^{\prime \prime} \geq q_{1}^{\prime} \geq k^{\prime} / k, k>k_{1}^{\prime}>0 \\
\left(k^{\prime}+1\right) / k>q_{1}^{\prime \prime} \geq q_{1}^{\prime}>k^{\prime} / k, k^{\prime}=0
\end{array}\right. \\
\text { We can obtain that: }\left\{\begin{array}{l}
{\left[k q_{1}^{\prime}\right]=\left[k q_{1}^{\prime \prime}\right]=k^{\prime}, k>k^{\prime}>0} \\
{\left[k q_{1}^{\prime}\right]=\left[k q_{1}^{\prime \prime}\right]=0, k^{\prime}=0}
\end{array} .\right.
\end{gathered}
$$

On the basis of $H_{q_{1}}^{\prime}(\theta)>0$, when $q_{1}=q_{1}^{\prime}, H_{q_{1}}\left(\theta_{1}\right)$ has the only equilibrium solution, which can be called $\theta_{q_{1}}$; when $q_{1}=q_{1}^{\prime \prime}, H_{q_{1}}\left(\theta_{1}\right)$ also has the only equilibrium solution, called $\theta_{q_{1}}$. For the equation $\left[k q_{1}^{\prime}\right]=\left[k q_{1}^{\prime \prime}\right]$ being permanent under both the environment of $k>k_{1}>0$ and $k_{1}=0$, equation $H_{q_{1}}\left(\theta_{1}\right)=H_{q_{i}}\left(\theta_{1}\right)$ is also tenable.

(ii) Effect of risk dominant degree in different interval

As for the risk dominant degree $q_{1}^{\prime}$ and $q_{1}^{\prime \prime}$ of the investment behavior "buy" in different interval, assuming that there is the following relationship between $q_{1}^{\prime}$ and $q_{1}^{\prime \prime}$. 


$$
\left\{\begin{array}{l}
q_{1}^{\prime \prime} \geq\left(k^{\prime}+1\right) / k>q_{1}^{\prime} \geq k^{\prime} / k, k>k^{\prime}>0 \\
q_{1}^{\prime \prime} \geq\left(k^{\prime}+1\right) / k>q_{1}^{\prime}>k^{\prime} / k, k^{\prime}=0
\end{array}\right.
$$

As the same above, we can obtain that $\left[k q_{1}^{\prime}\right]=k^{\prime}$ and $\left[k q_{1}^{\prime \prime}\right]=k^{\prime}+1$, and $\left[k q_{1}^{\prime}\right]<\left[k q_{1}^{\prime \prime}\right]$. Considering that the equilibrium solution of investment behavior "buy" in stable state can be set as follows:

$$
\begin{aligned}
& H_{q_{1}}\left(\theta_{q_{1}}\right)=\frac{\sum_{k \geq 1} k P(k)}{<k>} \sum_{k_{1}=k k q_{1}+1+1}^{k}\left(\begin{array}{l}
k \\
k_{1}
\end{array}\right) \theta_{1_{1}^{k_{1}}\left(1-\theta_{1}\right)^{k-k_{1}}} \\
& =\frac{\sum_{k \geq 1} k P(k)}{<k>} \sum_{k}^{k}\left(\begin{array}{l}
k \\
k_{1}
\end{array}\right) \theta_{1_{1}^{k_{1}}\left(1-\theta_{1}\right)^{k-k_{1}}} \\
& H_{q_{q_{1}}}\left(\theta_{q_{1}}\right)=\frac{\sum_{k \geq 1} k P(k)}{<k>} \sum_{\left.k_{1}=k k q_{1}\right]+1}^{k}\left(\begin{array}{l}
k \\
k_{1}
\end{array}\right) \theta_{1}^{k_{1}}\left(1-\theta_{1}\right)^{k-k_{1}} \\
& =\frac{\sum_{k \geq 1} k P(k)}{<k>} \sum_{k+1}^{k}\left(\begin{array}{l}
k \\
k_{1}
\end{array}\right) \theta_{1}^{k_{1}}\left(1-\theta_{1}\right)^{k-k_{1}}
\end{aligned}
$$

We can obtain the equation as follows:

$$
H_{q_{1}}\left(\theta_{q_{1}}\right)=H_{q_{1}^{\prime}}\left(\theta_{q_{1}}\right)+\frac{\sum_{k \geq 1}^{\prime} k P(k)}{<k>} \sum_{k^{\prime}}^{k^{\prime}+1}\left(\begin{array}{l}
k \\
k_{1}
\end{array}\right) \theta_{1}^{k_{1}}\left(1-\theta_{1}\right)^{k-k_{1}} .
$$

Thus, for the arbitrarily $\theta_{1}$, the equation $H_{q_{1}}\left(\theta_{q_{1}}\right)>H_{q_{1}}\left(\theta_{q_{1}}\right)$ is tenable. For the expression $H_{q_{1}}\left(\theta_{q_{1}}\right)$ and $H_{q_{1}}\left(\theta_{q_{1}}\right)$ both having the only equilibrium solution respectively, $H_{q_{1}}\left(\theta_{1}\right)$ decreases monotony as the decreasing of risk dominant degree in different intervals, and the logic relationship between $H_{q_{1}}\left(\theta_{1}\right)$ and $q_{1}$ can be seen in Fig. 1.

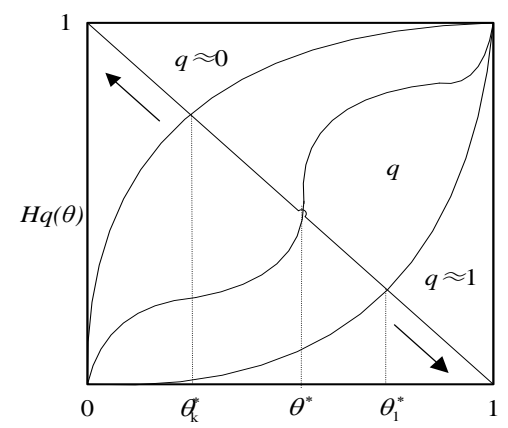

Fig.1. Logic Relationship between the equilibrium of investment behavior and risk dominance degree

\subsubsection{Simulation analysis}

Simulation analysis on the evolution of investment behavior's equilibrium state is conducted in this section. Let $N=500$ be the number of investor and $S_{N}=0$ be the initial state of investors' behavior, simulation experiment will be done 100 times and each of them will go 100 time steps. Degree $<k>=4$ and $<k>=12$ of ER Network [15], WS Network [16], BA Network [17] and IN Network [18] are comparatively analyzed in the simulation respectively. Notice that, this article focus on behavior "buy", we assume that the risk dominance degree of investment behavior "sell" and "hold" varies stochastically with the degree of behavior "buy", and the principle that risk dominance degree of investment behavior "sell" is lower than the degree of behavior "hold" is permanent. 



Fig. 2. Evolution of equilibrium of investment behavior with the risk dominance degree

Fig.2. depicts the evolution of equilibrium of investment behavior with the risk dominance degree under the strategy of coordination game. On the whole, the proportion of investors who select behavior "buy" declines as the growth of its risk dominance degree; while the proportion of investors who select behavior "sell" shows the opposite variation tendency. In the four kinds of network, the proportion of investors who select behavior "hold" basically tends to zero, and is almost not affected by the risk dominance degree. In Fig.2, the proportions of investors who select behavior "buy" and "sell" show both relatively stable when $q_{1} \in(0,0.2)$; while $q_{1} \in(0.2,1)$, the proportions present decreasing and increasing respectively. In addition, the proportions of investors who select behavior "buy" and "sell" within the same range fluctuate more severely in BA Network and IN Network than in ER Network and WS Network.

\subsection{Influence of network topology structure on the evolution of herding behavior}

In order to deconstruct the influence on the evolution of investment behavior in stock market from the network topology structure, this paper introduced the stochastic dominance theory to describe the network connectivity and heterogeneity, and analyzed the two factors' effect on the investment behavior. The first-order stochastic dominance is used to describe network connectivity, that is to say, if connectivity $<k>_{G_{1}}$ of $G_{1}$ is bigger than $<k>_{G_{1}}$ of $G_{2}, G_{1}$ first-order stochastically dominates $G_{2}$. Considering that network heterogeneity reflects variance characteristics of the degree of network connectivity, the second-order stochastic dominance is used to describe network's heterogeneity, that is to say, if $G_{2}$ 's heterogeneity is higher than $G_{1}$ 's, $G_{1}$ second-order stochastically dominates $G_{2}$. Analysis of influence on the stock market herding behavior from network connectivity degree and heterogeneity by the methods of numerical analysis and simulation respectively are as follows.

\subsubsection{Theoretical analysis}

( $\mathrm{i}$ ) Effect of network connectivity degree on the evolution of herding behavior

Let $P(k)$ and $P(k)$ be the degree distribution of network connectivity of two different network, and we assume that not only $P(k)$ first order dominance $P(k)$, but also $H_{q 1}^{\prime \prime}(\theta)<0$. Based on this, when $\theta=0$, there is one and only $[k q]=0(k \leq[1 / q])$, so we can value $k_{1}=1$ and get the expression as follows is permanent. 


$$
H_{q}^{\prime}(0)=\frac{1}{<k>} \sum_{k \geq 1}^{[1 / q]} k P(k)\left(\begin{array}{l}
k \\
1
\end{array}\right)=\frac{1}{<k>} \sum_{k \geq 1}^{[1 / q]} k^{2} P(k)>0 .
$$

Considering that $P(k)$ first order dominance $P(k)$ and $k^{2}$ is monotonically increasing in the range $k \in[0, \infty)$, based on the theory of first order dominance, we can make a conclusion that $\sum_{k \geq 1}^{k^{\prime}} k^{2} P(k) \geq \sum_{k \geq 1}^{k^{\prime}} k^{2} P(k)$. For the investment behavior "buy being risk dominant, we can get the conclusion that:

$$
H_{q_{1}}\left(\theta_{1}\right)=\frac{\sum_{k \geq 1} k P(k)}{<k>} \sum_{k_{1}=0}^{k} P_{q_{1}}\left(1 \mid k_{1}, k\right)\left(\begin{array}{c}
k \\
k_{1}
\end{array}\right) \theta_{1}^{k_{1}}\left(1-\theta_{1}\right)^{k-k_{1}} \geq H_{q_{1}}\left(\theta_{1}\right)=\frac{\sum_{k \geq 1} k P(k)}{<k>} \sum_{k_{1}=0}^{k} P_{q_{1}}\left(1 \mid k_{1}, k\right)\left(\begin{array}{c}
k \\
k_{1}
\end{array}\right) \theta_{1}^{k_{1}}\left(1-\theta_{1}\right)^{k-k_{1}},
$$

where $k^{\prime}=\left[1 / q_{1}\right]$, that is to say $\theta_{q_{1 p(k)}} \geq \theta_{q_{1 p(k)}}$.

Thus, for investment behavior "buy" being risk dominant, the bigger the network connectivity degree, and the more conductive for investors to select the corresponding investment behavior.

(ii ) Effect of network heterogeneity on the evolution of herding behavior

Let $P(k)$ and $P(k)$ be the degree distribution of network connectivity of two network different with different heterogeneity, and we assume that not only $P(k)$ second order dominance $P(k)$, but also $H_{q_{1}}^{\prime}(\theta)>0$ and $H_{q_{1}}^{\prime \prime}(\theta)<0$. According to the second order stochastic dominance theory, expression $\sum_{k \geq 1}^{k^{\prime}} k^{2} P(k) \geq \sum_{k \geq 1}^{k^{\prime}} k^{2} P(k)$ is concluded, where $0<k^{\prime}<k$. Thus, for the investment behavior "buy" being risk dominant, the higher the network heterogeneity, the more conductive for investors to select the corresponding investment behavior.

Based on this, influence on the evolution of the equilibrium of investment behavior from the network structure is directly affected by the risk dominance of corresponding investment behavior. Supposing that investment behavior "buy" is risk dominant, the greater network connectivity degree and the higher the network heterogeneity, the higher proportion of investors who selecting the behavior "buy".

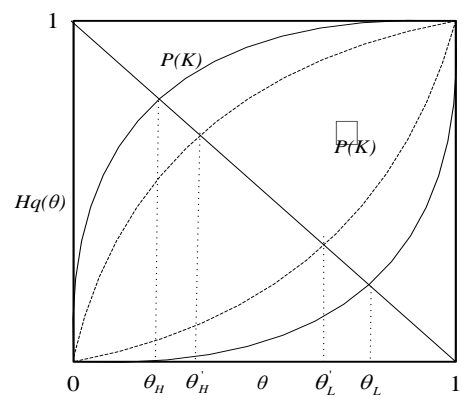

Fig.3. Logic Relationship between the equilibrium of some investment behavior being risk dominant and network characteristics

The logic relationship between the equilibrium of some kind of investment behavior being risk dominant and the network characteristics can be seen in Fig. 3. The solid convex line and the dotted convex line are the representatives of network connectivity degree distribution about two types of networks; while the solid concave line and the dotted concave line represent the difference of network heterogeneity. In the Figure, the equilibrium of investment behavior $\theta_{H}$ is larger than $\theta_{H}^{\prime}$ in two types of networks, where the distribution of network connectivity degree of the corresponding networks 
being $P(k)$ and $P(k)$, that $P(k)$ being first order dominance $P(k)$. The equilibrium of investment

behavior $\theta_{L}^{\prime}$ is larger than $\theta_{L}$, on the condition of the network heterogeneity, that $P(k)$ being second order dominance $P(k)$.

\subsubsection{Simulation analysis}

The experiment environment is the same as it in Section 4.1.2. Fig. 4 shows the evolution of the equilibrium of behavior "buy" in stock market as the risk dominance degree change. When $<k>=4$ or $<k>=10$, the equilibrium proportion of investment behavior "buy" decreases as its risk dominance degree increasing, and reflects approximate stepped down trend. Meanwhile, in the approximate interval $q_{1} \in(0,0.25)$ of risk dominance degree, the proportion of investors who select the behavior "buy" is relatively stable when the risk dominance degree of behavior "buy" is extremely high, that represents investors' behavior being consistent convergence. In ER network and WS network, the difference of network connectivity degree plays no influence on the equilibrium of investment behavior "buy". While in BA network and IN network, the proportion of investors' selecting behavior "buy" is higher when $\langle k\rangle=10$ than that when $\langle k\rangle=4$. That is to say, network connectivity degree $<k>=10$ relatively delays the decreasing speed of the proportion of behavior "buy", and the decreasing speed of the proportion of behavior "buy" as the risk dominance degree increasing represents more slowly. The simulation analysis result basically proofs the conclusion of numerical analysis.
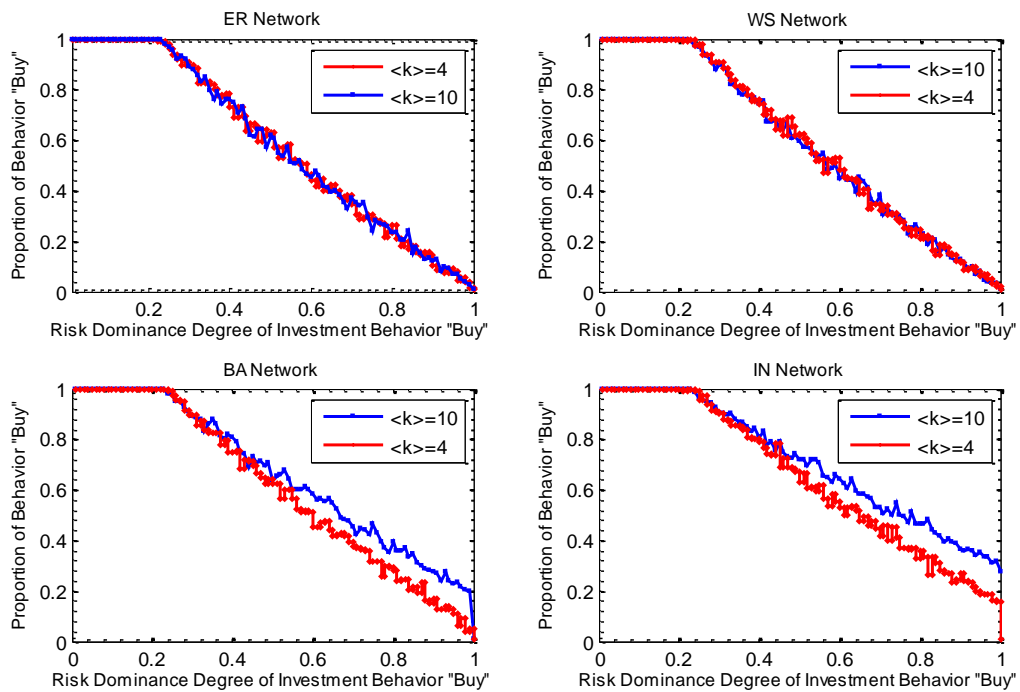

Fig. 4. Effect on the evolution of investment behavior in stock market from the difference of network connectivity degree, where

$$
<k>=4
$$

Fig. 5 and Fig. 6 both shows the influence on the evolution equilibrium of investment behavior "buy" from the heterogeneity of network. Fig. 5 shows that when $<k>=4$, the proportion of investment behavior "buy" increases as the decreasing of its risk dominance degree in all four type networks. The evolution equilibrium of the behavior "buy" in $B A$ network and $I N$ network is relatively higher than that in ER network and WS network, where the equilibrium in ER network and WS network is basically similar, which is highest in IN network. 




Fig. 5. Impact on the equilibrium of investment behavior from network heterogeneity and the comparative analysis in different networks, when $<k>=4$

In Fig.6, the evolution equilibrium state of the investment behavior "buy" is similar as it in Fig. 5. In addition, the decreasing speed of the evolution equilibrium of investment behavior is relatively slow, and the equilibrium proportion of the investors" selecting behavior "buy" is far more than in $E R$ network and WS network. Thus, when investment behavior "buy" is risk dominant, the bigger of network connectivity degree and the more heterogeneous of the network, the more advantageous for investors to select it.

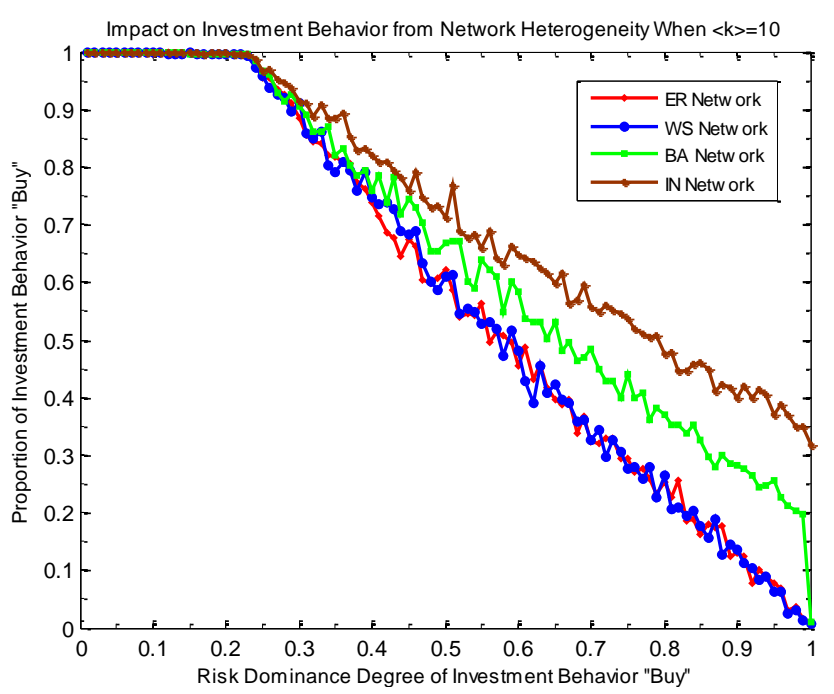

Fig. 6. Impact on the equilibrium of investment behavior from network heterogeneity and the comparative analysis in different networks, when $<k>=10$

\section{Conclusion}

In this paper, we introduce an evolution model of investors' trading behavior in stock market based on coordination game strategy in networks, according to the assumption of investors' limited rationality in behavioral financial literature. The model describes the evolution mechanism of investors' trading behavior from the equilibrium payoff matrix of coordination game. Let the behavior "buy" be the benchmark, we investigate by mean-field analysis and extensive simulations the evolution of investors' trading behavior in various typical networks under different characteristics of invest strategy in coordination game. Our results indicate that the evolution of investors' trading behavior in affected by the network structure of stock market and the risk dominant degree of investment behavior. Particularly, there exists ladder shaped non-strict decreasing between the equilibrium states of the proportion of 
investors' selecting some certain behavior and the risk dominance degree of corresponding investment behavior. Besides, if a certain investment behavior is risk dominant, the greater the connectivity degree and the higher the heterogeneity of the network, the more conductive to investors to selecting the corresponding investment behavior.

However, in this work, the evolution model is comparatively simple while that of the investors' trading behavior in real life is much more complex. Especially, investors' preference to certain trading behavior being deterministic and stochastic [19], and beliefs among investors' being heterogeneous [20], therefore, our future study will focus on the models that are even closer to the ones in real life, which could include considering the influence from the network structure based on social relationship, online network and other relationship among investors, including the co-evolution between investment behavior and network structure under different evolution mechanism.

\section{Acknowledgment}

We wish to thank the anonymous referee for his/her invaluable comments and suggestions. This research was supported by the National Natural Science Foundation of China (Grant No. 71301078, 71372181, and 71201023), Project supported by the Natural Science Foundation of Education Bureau of Jiangsu Province (Grant No. 13KJB120006 and 2014SJB094) and The National Science Foundation for Post-doctoral Scientists of China (Grant No. 2014M551623).

\section{References}

[1] Villatoro, F., The delegated portfolio management problem: reputation and herding. Journal of Banking Finance 33 (2009) 2062-2069.

[2] Nofsinger, J., Sias, R., Herding and feedback trading by institutional and individual investors. Journal of Finance 54 (1999) 2263-2295.

[3] Griffin, J. M., Harris, J. H., \& Topaloglu, S, The dynamics of institutional and individual trading. Journal of Finance 58 (2003) 2285-2320.

[4] Zhou, R., Lai, R., Herding and information based trading. Journal of Empirical Finance 16 (2009) 388-393.

[5] Chiang, T. C., D. Z., Zheng., An empirical analysis of herd behavior in global stock markets. Journal of Banking \& Finance 34 (2010) 1911-1921.

[6] Demirer, R., Kutan, A. M., 2006. Does herding behavior exist in Chinese stock markets? Journal of International Financial Markets Institutions and Money 16, 123-142.

[7] Tan, L., Chiang, T. C., Mason, J. R., \& Nelling, E., Herding behavior in Chinese stock markets: An examination of A and B shares. Pacific-Basin Finance Journal 16 (2008) 61-77.

[8] Y. Wei, S. Ying, Y. Fan, B.-H. Wang, The cellular automaton model of investment behavior in the stock market, Physica A 325 (2003) 507-516.

[9] Z. Z. Liang, Q. L. Han, Coherent artificial stock market model based on small world networks, Complex Systems and Complexity Science, 2 (2009) 70-76 (in Chinese)

[10] L. Bakker, W. Hare, H. Khosravi, B. Ramadanovic, A social network model of investment behavior in the stock market, physica A 389 (2010) 1223-1229.

[11] Y. Chen, J. H. Yuan, X. D. Li, et al., Research on collaborative herding behavior and market volatility: Based on computational experiments. Journal of Management Sciences in China, 13 (2010) 119-128.

[12] P. Falbo, R. Grassi. Market Dynamics When Agents Anticipate Correlation Breakdown, Discrete Dynamics in Nature and Society, 2011, doi:10.1155/2011/959847.

[13] Johansen A, Sornette D., Log-periodic power law bubbles in Latin-American and Asian markets and correlated anti-bubbles in Western stock markets-An empirical study. International Journal of Theoretical and Applied Finance, 4 (2001) 853-920.

[14] Albert R, Barabási A L., Statistical mechanics of complex networks. Rev. Mod. Phys, 74 (2002) 47-97.

[15] Erdös P, Rényi A. On the evolution of random graphs. Publ. Math. Inst. Hung. Acad. Sci., 1960, 5: 17-60.

[16] Watts D J, Strogatz S H. Collective dynamics of 'small-world' networks. Nature, 1998, 393: 440-442.

[17] Barabási A-L, Albert R. Emergence of scaling in random networks. Science, 1999, 286: 509-512.

[18] Y. T. Bian, J. M. He, Y. M. Zhuang, A Network Model of Investment and Its Robustness Based on the Intrinsic Characteristics of the Subjects in Stock Market. Journal of Industrial Engineering and Engineering Management, 2013, 108-113.

[19] L. I. Dobrescu, M. Neamtu, A. L. Ciurdariu, et al. A Dynamic Economic Model with Discrete Time and Consumer Sentiment, Discrete Dynamics in Nature and Society, 2009, doi:10.1155/2009/509561.

[20] A. Foster, N. Kirby. Analysis of a Heterogeneous Trader Model for Asset Price Dynamics, Discrete Dynamics in Nature and Society, 2011, doi:10.1155/2011/309572. 